\title{
Stress Life Analysis of Induction Furnace Wall for Magnesia Ramming Mass
}

\author{
Prof. Nirajkumar C Mehta ${ }^{1}$, Dr. Dipesh D Shukla ${ }^{2}$ \\ ${ }^{I}$ Ph. D. Student, Rai University, Ahmedabad, India \\ ${ }^{2}$ Director, Amity University, Jaipur, India
}

\begin{abstract}
Furnaces are most commonly used for melting of materials. Induction furnaces are more beneficial as no fuel is required. It is a problem to find life cycle of Induction Melting Furnace Wall under load variation. The induction melting furnace wall is made of magnesia ramming mass which is one kind of refractory material. The failure occurs due to cyclic thermal stresses due to heating and cooling cycles. Temperature distribution and thermal stress distribution fields of the induction melting furnace refractory wall were calculated by using explicit finite difference analysis based on the physical description of its failure under low cycle thermal fatigue conditions. The life span of the refractory wall is required to be found out by means of critical thermal stresses created inside the refractory wall of induction melting furnace wall from modified $S-\log N$ Curve.
\end{abstract}

Keywords: Advanced stress life analysis, Temperature distribution, Stress distribution, explicit finite difference method, Magnesia ramming mass

\section{Introduction}

Furnace is a term used to identify a closed space here heat is applied to a body in order to raise its temperature. The source of heat may be fuel or electricity. Commonly, metals and alloys and sometimes nonmetals are heated in furnaces. The purpose of heating defines the temperature of heating and heating rate. Increase in temperature softens the metals. They become amenable to deformation. This softening occurs with or without a change in the metallic structure. Heating to lower temperatures (below the critical temperature) of the metal softens it by relieving the internal stresses. On the other hand, metals heated to temperatures above the critical temperatures leads to changes in crystal structures and recrystallization like annealing. Further some metals and alloys are melted, ceramic products vitrified, coals coked, metals like zinc are vaporized and many other processes are performed in Furnaces.

Induction furnaces are widely used in the iron industry for the casting of the different grades of cast iron products. Refractory wall of induction melting furnace is a key component which is used as insulation layer. It is made of ramming mass like silica, alumina, magnesia etc. The refractory wall is directly influenced by the thermal cycling of the high temperature molten iron in the furnace. Thermal fatigue failure is easy to happen for it because of the larger phase transformation thermal stresses and it has a shorter life. This can cause serious production accidents. Therefore, the service life problem of the refractory wall has always been a focus of attention in the application of this to the industry. (A V K Suryanarayana, Fuels Furnaces Refractory and Pyrometry).

A comprehensive literature review on computational investigation on different kinds of furnaces is done to study research trends. (Nirajkumar Mehta, May 2012). A review is done on applications of different numerical methods in heat transfer with its applications. (N C Mehta, Vipul B Gondaliya et al, February 2013). Thermal fatigue analysis of induction melting furnace wall is done for silica ramming mass. (N C Mehta, Akash D Raiyani et al, February 2013). A review is done for research on induction heating. (Vimal R Nakum et al, April 2013). A review is done for metal forming analysis using different numerical methods. (N C Mehta et al, May 2013). Transient heat transfer analysis of induction furnace is done by using finite element analysis. (Vipul Gondaliya et al, August 2013). Thermal fatigue analysis of induction furnace wall is done for alumina ramming mass. (N C Mehta et al, October 2013).

Thermal analysis of hot wall condenser is done for domestic refrigerator using numerical method for temperature distribution. (Akash D Raiyani et al, July 2014). Optimisation of wall thickness is done for minimum heat loss for induction furnace by finite element analysis. (Dipesh D Shukla et al, December 2014). A review is done on numerical analysis of furnace. (N C Mehta et al, April 2015). Thermal fatigue analysis of induction furnace wall is done for zirconia. (Nirajkumar C Mehta et al, April 2015). Comparison of finite difference method and finite element method is done for $2 \mathrm{D}$ transient heat transfer problem. (Nirajkumar Mehta et al, April 2015). Thermal fatigue analysis of induction furnace wall is done for magnesia ramming mass. (Nirajkumar C Mehta et al, June 2015). Advanced mathematical modelling of heat transfer is done for induction furnace wall of zirconia. (Nirajkumar C Mehta et al, December 2016). Advanced heat transfer analysis is done for magnesia based refractory wall of induction furnace. (Nirajkumar Mehta et al, December 2016). 
Here, Explicit Finite Difference Method is used to find out temperature and thermal stress variation with respect to time.

\section{Development of Advanced Explicit Finite Difference Model}

We have divided Induction Furnace Wall into a Nodal Network as shown in Fig. 1. It is divided into 24 nodes. We have derived Explicit Finite Difference Equations for all nodes as per the boundary conditions applied to it. The furnace wall is having thermal conduction heat transfer between different nodes. It is having atmospheric heat convection ha applied from top side of the furnace wall which is open to atmosphere. It is having heat convection from molten metal from inside which is hi. It is having heat convection ho from cooling water which is circulating outside the furnace wall. (Yunus A Cengel, Heat and Mass Transfer).

To solve this advanced heat transfer problem of induction melting furnace wall which is made from Magnesia Ramming Mass, the following initial and boundary conditions, material properties and basic assumptions are made:

Refractory Materials for induction furnace wall meets the basic assumptions in the science of mechanics.

$>$ Environmental Temperature is homogeneous at $27^{\circ} \mathrm{C}$.

$>$ Ignore the influence of heat radiation.

$>$ Ignore the effect of gravity field.

$>$ The surface of induction melting furnace wall is clean.

$>$ The initial temperature of the induction melting furnace is set $27^{\circ} \mathrm{C}$ and it is agreement with the ambient temperature during solving the problem.

$>$ Heat convections are considered constant for this analysis.

$>$ Scarp material input inside furnace is considered uniform for our analysis.

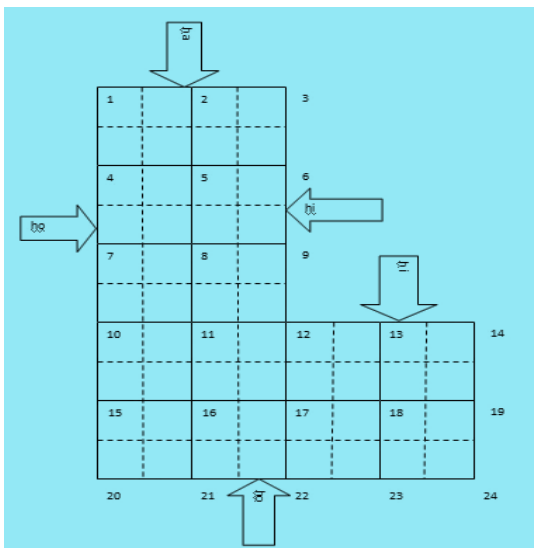

Fig.1 Nodal network for finite difference method

Node 1:

$h a \frac{\Delta x}{2}\left(T_{\infty}-T_{1}^{i}\right)+h o \frac{\Delta y}{2}\left(T_{\infty}-T_{1}^{i}\right)+\mathrm{k} \frac{\Delta y}{2} \frac{T_{2}^{i}-T_{1}^{i}}{\Delta x}+\mathrm{k} \frac{\Delta x}{2} \frac{T_{4}^{i}-T_{1}^{i}}{\Delta y}=\rho \frac{\Delta x}{2} \frac{\Delta y}{2} \mathrm{C} \frac{T_{1}^{i+1}-T_{1}^{i}}{\Delta t}$

$T_{1}^{i+1}=\left(\left(h a \frac{\Delta x}{2}\left(T_{\infty}-T_{1}^{i}\right)+h o \frac{\Delta y}{2}\left(T_{\infty}-T_{1}^{i}\right)+\mathrm{k} \frac{\Delta y}{2} \frac{T_{2}^{i}-T_{1}^{i}}{\Delta x}+\mathrm{k} \frac{\Delta x}{2} \frac{T_{4}^{i}-T_{1}^{i}}{\Delta y}\right) \frac{4 \Delta t}{\rho C \Delta x \Delta y}\right)+T_{1}^{i}$

Node 2:

$h a \Delta x\left(T_{\infty}-T_{2}^{i}\right)+\mathrm{k} \frac{\Delta y}{2} \frac{T_{1}^{i}-T_{2}^{i}}{\Delta x}+\mathrm{k} \frac{\Delta y}{2} \frac{T_{3}^{i}-T_{2}^{i}}{\Delta x}+\mathrm{k} \Delta x \frac{T_{5}^{i}-T_{2}^{i}}{\Delta y}=\rho \Delta x \frac{\Delta y}{2} \mathrm{C} \frac{T_{2}^{i+1}-T_{2}^{i}}{\Delta t}$

$T_{2}^{i+1}=\left(\left(h a \Delta x\left(T_{\infty}-T_{2}^{i}\right)+\mathrm{k} \frac{\Delta y}{2} \frac{T_{1}^{i}-T_{2}^{i}}{\Delta x}+\mathrm{k} \frac{\Delta y}{2} \frac{T_{3}^{i}-T_{2}^{i}}{\Delta x}+\mathrm{k} \Delta x \frac{T_{5}^{i}-T_{2}^{i}}{\Delta y}\right) \frac{2 \Delta t}{\rho C \Delta x \Delta y}\right)+T_{2}^{i}$

Node 3:

$h a \frac{\Delta x}{2}\left(T_{\infty}-T_{3}^{i}\right)+h i \frac{\Delta y}{2}\left(T_{h}-T_{3}^{i}\right)+\mathrm{k} \frac{\Delta y}{2} \frac{T_{2}^{i}-T_{3}^{i}}{\Delta x}+\mathrm{k} \frac{\Delta x}{2} \frac{T_{6}^{i}-T_{3}^{i}}{\Delta y}=\rho \frac{\Delta x}{2} \frac{\Delta y}{2} \mathrm{C} \frac{T_{3}^{i+1}-T_{3}^{i}}{\Delta t}$

$T_{3}^{i+1}=\left(\left(h a \frac{\Delta x}{2}\left(T_{\infty}-T_{3}^{i}\right)+h i \frac{\Delta y}{2}\left(T_{h}-T_{3}^{i}\right)+\mathrm{k} \frac{\Delta y}{2} \frac{T_{2}^{i}-T_{3}^{i}}{\Delta x}+\mathrm{k} \frac{\Delta x}{2} \frac{T_{6}^{i}-T_{3}^{i}}{\Delta y}\right) \frac{4 \Delta t}{\rho C \Delta x \Delta y}\right)+T_{3}^{i}$

Node 4:

$h o \Delta y\left(T_{\infty}-T_{4}^{i}\right)+\mathrm{k} \Delta y \frac{T_{5}^{i}-T_{4}^{i}}{\Delta x}+\mathrm{k} \frac{\Delta x}{2} \frac{T_{1}^{i}-T_{4}^{i}}{\Delta y}+\mathrm{k} \frac{\Delta x}{2} \frac{T_{7}^{i}-T_{4}^{i}}{\Delta y}=\rho \frac{\Delta x}{2} \Delta y C \frac{T_{4}^{i+4}-T_{4}^{i}}{\Delta t}$ 
$T_{4}^{i+4}=\left(\left(h \circ \Delta y\left(T_{\infty}-T_{4}^{i}\right)+\mathrm{k} \Delta y \frac{T_{5}^{i}-T_{4}^{i}}{\Delta x}+\mathrm{k} \frac{\Delta x}{2} \frac{T_{1}^{i}-T_{4}^{i}}{\Delta y}+\mathrm{k} \frac{\Delta x}{2} \frac{T_{7}^{i}-T_{4}^{i}}{\Delta y}\right) \frac{2 \Delta t}{\rho(\Delta x \Delta y}\right)+T_{4}^{i}$

Node 5:

$\mathrm{k} \Delta y \frac{T_{4}^{i}-T_{5}^{i}}{\Delta x}+\mathrm{k} \Delta y \frac{T_{6}^{i}-T_{5}^{i}}{\Delta x}+\mathrm{k} \Delta x \frac{T_{2}^{i}-T_{5}^{i}}{\Delta y}+\mathrm{k} \Delta x \frac{T_{\frac{i}{i}-T_{5}^{i}}^{i}}{\Delta y}=\rho \Delta x \Delta y C \frac{C_{5}^{i+1}-T_{5}^{i}}{\Delta t}$

$T_{5}^{i+1}=\left(\left(\mathrm{k} \Delta y \frac{T_{4}^{i}-T_{5}^{i}}{\Delta x}+\mathrm{k} \Delta y \frac{T_{6}^{i}-T_{5}^{i}}{\Delta x}+\mathrm{k} \Delta x \frac{T_{2}^{i}-T_{5}^{i}}{\Delta y}+\mathrm{k} \Delta x \frac{T_{8}^{i}-T_{5}^{i}}{\Delta y}\right) \frac{\Delta t}{\rho C \Delta x \Delta y}\right)+T_{5}^{i}$

Node 6:

$h i \Delta y\left(T_{h}-T_{6}^{i}\right)+\mathrm{k} \Delta y \frac{T_{2}^{i}-T_{6}^{i}}{\Delta x}+\mathrm{k} \frac{\Delta x}{2} \frac{T_{3}^{i}-T_{6}^{i}}{\Delta y}+\mathrm{k} \frac{\Delta x}{2} \frac{T_{9}^{i}-T_{6}^{i}}{\Delta y}=\rho \frac{\Delta x}{2} \Delta y C \frac{T_{6}^{i+1}-T_{6}^{i}}{\Delta t}$

$T_{6}^{i+1}=\left(\left(h i \Delta y\left(T_{h}-T_{6}^{i}\right)+\mathrm{k} \Delta y \frac{T_{2}^{i}-T_{6}^{i}}{\Delta x}+\mathrm{k} \frac{\Delta x}{2} \frac{T_{3}^{i}-T_{6}^{i}}{\Delta y}+\mathrm{k} \frac{\Delta x}{2} \frac{T_{9}^{i}-T_{6}^{i}}{\Delta y}\right) \frac{2 \Delta t}{\rho(\Delta x \Delta y}\right)+T_{6}^{i}$

Node 7:

$h o \Delta y\left(T_{\infty}-T_{7}^{i}\right)+\mathrm{k} \Delta y \frac{T_{8}^{i}-T_{7}^{i}}{\Delta x}+\mathrm{k} \frac{\Delta x}{2} \frac{T_{4}^{i}-T_{7}^{i}}{\Delta y}+\mathrm{k} \frac{\Delta x}{2} \frac{T_{10}^{i}-T_{7}^{i}}{\Delta y}=\rho \frac{\Delta x}{2} \Delta y C \frac{T_{7}^{i+7}-T_{7}^{i}}{\Delta t}$

$T_{7}^{i+7}=\left(\left(h \circ \Delta y\left(T_{\infty}-T_{7}^{i}\right)+\mathrm{k} \Delta y \frac{T_{8}^{i}-T_{7}^{i}}{\Delta x}+\mathrm{k} \frac{\Delta x}{2} \frac{T_{4}^{i}-T_{7}^{i}}{\Delta y}+\mathrm{k} \frac{\Delta x}{2} \frac{T_{10}^{i}-T_{7}^{i}}{\Delta y}\right) \frac{2 \Delta t}{\rho C \Delta x \Delta y}\right)+T_{7}^{i}$

Node 8:

$\mathrm{k} \Delta y \frac{T_{7}^{i}-T_{8}^{i}}{\Delta x}+\mathrm{k} \Delta y \frac{T_{9}^{i}-T_{8}^{i}}{\Delta x}+\mathrm{k} \Delta x \frac{T_{5}^{i}-T_{8}^{i}}{\Delta y}+\mathrm{k} \Delta x \frac{T_{11}^{i}-T_{8}^{i}}{\Delta y}=\rho \Delta x \Delta y \mathrm{C} \frac{T_{8}^{i+1}-T_{8}^{i}}{\Delta t}$

$T_{8}^{i+1}=\left(\left(\mathrm{k} \Delta y \frac{T_{7}^{i}-T_{8}^{i}}{\Delta x}+\mathrm{k} \Delta y \frac{T_{9}^{i}-T_{8}^{i}}{\Delta x}+\mathrm{k} \Delta x \frac{T_{5}^{i}-T_{8}^{i}}{\Delta y}+\mathrm{k} \Delta x \frac{T_{11}^{i}-T_{8}^{i}}{\Delta y}\right) \frac{\Delta t}{\rho C \Delta x \Delta y}\right)+T_{8}^{i}$

Node 9:

$h i \Delta y\left(T_{h}-T_{9}^{i}\right)+\mathrm{k} \Delta y \frac{T_{8}^{i}-T_{9}^{i}}{\Delta x}+\mathrm{k} \frac{\Delta x}{2} \frac{T_{-}^{i}-T_{9}^{i}}{\Delta y}+\mathrm{k} \frac{\Delta x}{2} \frac{T_{2}^{i}-T_{9}^{i}}{\Delta y}=\rho \frac{\Delta x}{2} \Delta y C \frac{T_{9}^{i+1}-T_{9}^{i}}{\Delta t}$

$T_{9}^{i+1}=\left(\left(h i \Delta y\left(T_{h}-T_{9}^{i}\right)+\mathrm{k} \Delta y \frac{T_{8}^{i}-T_{9}^{i}}{\Delta x}+\mathrm{k} \frac{\Delta x}{2} \frac{T_{6}^{i}-T_{9}^{i}}{\Delta y}+\mathrm{k} \frac{\Delta x}{2} \frac{T_{12}^{i}-T_{9}^{i}}{\Delta y}\right) \frac{2 \Delta t}{\rho(\Delta x \Delta y}\right)+T_{9}^{i}$

Node 10:

$h o \Delta y\left(T_{\infty}-T_{10}^{i}\right)+\mathrm{k} \Delta y \frac{T_{11}^{i}-T_{10}^{i}}{\Delta x}+\mathrm{k} \frac{\Delta x}{2} \frac{T_{7}^{i}-T_{10}^{i}}{\Delta y}+\mathrm{k} \frac{\Delta x}{2} \frac{T_{15}^{i}-T_{10}^{i}}{\Delta y}=\rho \frac{\Delta x}{2} \Delta y C \frac{T_{10}^{i+10}-T_{10}^{i}}{\Delta t}$

$T_{10}^{i+10}=\left(\left(h o \Delta y\left(T_{\infty}-T_{10}^{i}\right)+\mathrm{k} \Delta y \frac{T_{11}^{i}-T_{10}^{i}}{\Delta x}+\mathrm{k} \frac{\Delta x}{2} \frac{T_{7}^{i}-T_{10}^{i}}{\Delta y}+\mathrm{k} \frac{\Delta x}{2} \frac{T_{15}^{i}-T_{10}^{i}}{\Delta y}\right) \frac{2 \Delta t}{\rho C \Delta x \Delta y}\right)+T_{10}^{i}$

Node 11:

$\mathrm{k} \Delta y \frac{T_{10}^{i}-T_{11}^{i}}{\Delta x}+\mathrm{k} \Delta y \frac{T_{12}^{i}-T_{11}^{i}}{\Delta x}+\mathrm{k} \Delta x \frac{T_{8}^{i}-T_{11}^{i}}{\Delta y}+\mathrm{k} \Delta x \frac{T_{16}^{i}-T_{11}^{i}}{\Delta y}=\rho \Delta x \Delta y \mathrm{C} \frac{T_{11}^{i+1}-T_{11}^{i}}{\Delta t}$

$T_{11}^{i+1}=\left(\left(\mathrm{k} \Delta y \frac{T_{10}^{i}-T_{11}^{i}}{\Delta x}+\mathrm{k} \Delta y \frac{T_{12}^{i}-T_{11}^{i}}{\Delta x}+\mathrm{k} \Delta x \frac{T_{8}^{i}-T_{11}^{i}}{\Delta y}+\mathrm{k} \Delta x \frac{T_{16}^{i}-T_{11}^{i}}{\Delta y}\right) \frac{\Delta t}{\rho C \Delta x \Delta y}\right)+T_{11}^{i}$

Node 12:

$h i \frac{\Delta x}{2}\left(T_{h}-T_{12}^{i}\right)+h i \frac{\Delta y}{2}\left(T_{h}-T_{12}^{i}\right)+\mathrm{k} \Delta y \frac{T_{11}^{i}-T_{12}^{i}}{\Delta x}+\mathrm{k} \frac{\Delta y}{2} \frac{T_{13}^{i}-T_{12}^{i}}{\Delta x}+\mathrm{k} \frac{\Delta x}{2} \frac{T_{9}^{i}-T_{12}^{i}}{\Delta y}+\mathrm{k} \Delta x \frac{T_{17}^{i}-T_{12}^{i}}{\Delta y}=\rho \frac{3 \Delta x \Delta y}{4} \mathrm{C} \frac{T_{12}^{i+1}-T_{12}^{i}}{\Delta t}$

$T_{12}^{i+1}=\left(\left(h i \frac{\Delta x}{2}\left(T_{h}-T_{12}^{i}\right)+h i \frac{\Delta y}{2}\left(T_{h}-T_{12}^{i}\right)+\mathrm{k} \Delta y \frac{T_{11}^{i}-T_{12}^{i}}{\Delta x}+\mathrm{k} \frac{\Delta y}{2} \frac{T_{13}^{i}-T_{12}^{i}}{\Delta x}+\mathrm{k} \frac{\Delta x}{2} \frac{T_{9}^{i}-T_{12}^{i}}{\Delta y}+\right.\right.$ $\left.\left.\mathrm{k} \Delta x \frac{T_{17}^{i}-T_{12}^{i}}{\Delta y}\right) \frac{4 \Delta t}{3 \rho(\Delta x \Delta y}\right)+T_{12}^{i}$

Node 13:

$h i \Delta x\left(T_{h}-T_{13}^{i}\right)+\mathrm{k} \frac{\Delta y}{2} \frac{T_{12}^{i}-T_{13}^{i}}{\Delta x}+\mathrm{k} \frac{\Delta y}{2} \frac{T_{4}^{i}-T_{13}^{i}}{\Delta x}+\mathrm{k} \Delta x \frac{T_{18}^{i}-T_{13}^{i}}{\Delta y}=\rho \Delta x \frac{\Delta y}{2} C \frac{T_{13}^{i+1}-T_{13}^{i}}{\Delta t}$

$T_{13}^{i+1}=\left(\left(h i \Delta x\left(T_{h}-T_{13}^{i}\right)+\mathrm{k} \frac{\Delta y}{2} \frac{T_{12}^{i}-T_{13}^{i}}{\Delta x}+\mathrm{k} \frac{\Delta y}{2} \frac{T_{14}^{i}-T_{13}^{i}}{\Delta x}+\mathrm{k} \Delta x \frac{T_{18}^{i}-T_{13}^{i}}{\Delta y}\right) \frac{2 \Delta t}{\rho(\Delta x \Delta y}\right)+T_{13}^{i}$

Node 14:

$h i \frac{\Delta x}{2}\left(T_{h}-T_{14}^{i}\right)+h i \frac{\Delta y}{2}\left(T_{h}-T_{14}^{i}\right)+\mathrm{k} \frac{\Delta y}{2} \frac{T_{13}^{i}-T_{14}^{i}}{\Delta x}+\mathrm{k} \frac{\Delta x}{2} \frac{T_{19}^{i}-T_{14}^{i}}{\Delta y}=\rho \frac{\Delta x}{2} \frac{\Delta y}{2} C \frac{T_{14}^{i+1}-T_{14}^{i}}{\Delta t}$ 
$T_{14}^{i+1}=\left(\left(h i \frac{\Delta x}{2}\left(T_{h}-T_{14}^{i}\right)+h i \frac{\Delta y}{2}\left(T_{h}-T_{14}^{i}\right)+\mathrm{k} \frac{\Delta y}{2} \frac{T_{13}^{i}-T_{14}^{i}}{\Delta x}+\mathrm{k} \frac{\Delta x}{2} \frac{T_{19}^{i}-T_{14}^{i}}{\Delta y}\right) \frac{4 \Delta t}{\rho(\Delta x \Delta y}\right)+T_{14}^{i}$

Node 15:

$h o \Delta y\left(T_{\infty}-T_{15}^{i}\right)+\mathrm{k} \Delta y \frac{T_{16}^{i}-T_{15}^{i}}{\Delta x}+\mathrm{k} \frac{\Delta x}{2} \frac{T_{10}^{i}-T_{15}^{i}}{\Delta y}+\mathrm{k} \frac{\Delta x}{2} \frac{T_{20}^{i}-T_{15}^{i}}{\Delta y}=\rho \frac{\Delta x}{2} \Delta y C \frac{T_{15}^{i+15}-T_{15}^{i}}{\Delta t}$

$T_{15}^{i+15}=\left(\left(h o \Delta y\left(T_{\infty}-T_{15}^{i}\right)+\mathrm{k} \Delta y \frac{T_{16}^{i}-T_{15}^{i}}{\Delta x}+\mathrm{k} \frac{\Delta x}{2} \frac{T_{10}^{i}-T_{15}^{i}}{\Delta y}+\mathrm{k} \frac{\Delta x}{2} \frac{T_{20}^{i}-T_{15}^{i}}{\Delta y}\right) \frac{2 \Delta t}{\rho C \Delta x \Delta y}\right)+T_{15}^{i}$

Node 16:

$\mathrm{k} \Delta y \frac{T_{15}^{i}-T_{16}^{i}}{\Delta x}+\mathrm{k} \Delta y \frac{T_{17}^{i}-T_{16}^{i}}{\Delta x}+\mathrm{k} \Delta x \frac{T_{11}^{i}-T_{16}^{i}}{\Delta y}+\mathrm{k} \Delta x \frac{T_{21}^{i}-T_{16}^{i}}{\Delta y}=\rho \Delta x \Delta y \mathrm{C} \frac{T_{16}^{i+1}-T_{16}^{i}}{\Delta t}$

$T_{16}^{i+1}=\left(\left(\mathrm{k} \Delta y \frac{T_{15}^{i}-T_{16}^{i}}{\Delta x}+\mathrm{k} \Delta y \frac{T_{17}^{i}-T_{16}^{i}}{\Delta x}+\mathrm{k} \Delta x \frac{T_{11}^{i}-T_{16}^{i}}{\Delta y}+\mathrm{k} \Delta x \frac{T_{21}^{i}-T_{16}^{i}}{\Delta y}\right) \frac{\Delta t}{\rho(\Delta x \Delta y}\right)+T_{16}^{i}$

Node 17:

$\mathrm{k} \Delta y \frac{T_{16}^{i}-T_{17}^{i}}{\Delta x}+\mathrm{k} \Delta y \frac{T_{18}^{i}-T_{17}^{i}}{\Delta x}+\mathrm{k} \Delta x \frac{T_{12}^{i}-T_{17}^{i}}{\Delta y}+\mathrm{k} \Delta x \frac{T_{22}^{i}-T_{17}^{i}}{\Delta y}=\rho \Delta x \Delta y \mathrm{C} \frac{T_{17}^{i+1}-T_{17}^{i}}{\Delta t}$

$T_{17}^{i+1}=\left(\left(\mathrm{k} \Delta y \frac{T_{16}^{i}-T_{17}^{i}}{\Delta x}+\mathrm{k} \Delta y \frac{T_{18}^{i}-T_{17}^{i}}{\Delta x}+\mathrm{k} \Delta x \frac{T_{12}^{i}-T_{17}^{i}}{\Delta y}+\mathrm{k} \Delta x \frac{T_{22}^{i}-T_{17}^{i}}{\Delta y}\right) \frac{\Delta t}{\rho(\Delta x \Delta y}\right)+T_{17}^{i}$

Node 18:

$\mathrm{k} \Delta y \frac{T_{17}^{i}-T_{18}^{i}}{\Delta x}+\mathrm{k} \Delta y \frac{T_{19}^{i}-T_{18}^{i}}{\Delta x}+\mathrm{k} \Delta x \frac{T_{13}^{i}-T_{18}^{i}}{\Delta y}+\mathrm{k} \Delta x \frac{T_{23}^{i}-T_{18}^{i}}{\Delta y}=\rho \Delta x \Delta y \mathrm{C}_{\frac{T_{18}^{i+1}-T_{18}^{i}}{\Delta t}}$

$T_{18}^{i+1}=\left(\left(\mathrm{k} \Delta y \frac{T_{17}^{i}-T_{18}^{i}}{\Delta x}+\mathrm{k} \Delta y \frac{T_{19}^{i}-T_{18}^{i}}{\Delta x}+\mathrm{k} \Delta x \frac{T_{13}^{i}-T_{18}^{i}}{\Delta y}+\mathrm{k} \Delta x \frac{T_{23}^{i}-T_{18}^{i}}{\Delta y}\right) \frac{\Delta t}{\rho(\Delta x \Delta y}\right)+T_{18}^{i}$

Node 19:

$h i \Delta y\left(T_{h}-T_{19}^{i}\right)+\mathrm{k} \Delta y \frac{T_{18}^{i}-T_{19}^{i}}{\Delta x}+\mathrm{k} \frac{\Delta x}{2} \frac{T_{14}^{i}-T_{19}^{i}}{\Delta y}+\mathrm{k} \frac{\Delta x}{2} \frac{T_{24}^{i}-T_{19}^{i}}{\Delta y}=\rho \frac{\Delta x}{2} \Delta y C_{19}^{T_{19}^{i+1}-T_{19}^{i}}$

$T_{19}^{i+1}=\left(\left(h i \Delta y\left(T_{h}-T_{19}^{i}\right)+\mathrm{k} \Delta y \frac{T_{18}^{i}-T_{19}^{i}}{\Delta x}+\mathrm{k} \frac{\Delta x}{2} \frac{T_{14}^{i}-T_{19}^{i}}{\Delta y}+\mathrm{k} \frac{\Delta x}{2} \frac{T_{24}^{i}-T_{19}^{i}}{\Delta y}\right) \frac{2 \Delta t}{\rho(\Delta x \Delta y}\right)+T_{19}^{i}$

Node 20:

$h o \frac{\Delta x}{2}\left(T_{\infty}-T_{20}^{i}\right)+h o \frac{\Delta y}{2}\left(T_{\infty}-T_{20}^{i}\right)+\mathrm{k} \frac{\Delta y}{2} \frac{T_{21}^{i}-T_{20}^{i}}{\Delta x}+\mathrm{k} \frac{\Delta x}{2} \frac{T_{15}^{i}-T_{20}^{i}}{\Delta y}=\rho \frac{\Delta x}{2} \frac{\Delta y}{2} C \frac{T_{20}^{i+20}-T_{20}^{i}}{\Delta t}$

$T_{20}^{i+20}=\left(\left(h o \frac{\Delta x}{2}\left(T_{\infty}-T_{20}^{i}\right)+h o \frac{\Delta y}{2}\left(T_{\infty}-T_{20}^{i}\right)+\mathrm{k} \frac{\Delta y}{2} \frac{T_{21}^{i}-T_{20}^{i}}{\Delta x}+\mathrm{k} \frac{\Delta x}{2} \frac{T_{15}^{i}-T_{20}^{i}}{\Delta y}\right) \frac{4 \Delta t}{\rho C \Delta x \Delta y}\right)+T_{20}^{i}$

Node 21:

$h o \Delta x\left(T_{\infty}-T_{21}^{i}\right)+\mathrm{k} \frac{\Delta y}{2} \frac{T_{0}^{i}-T_{21}^{i}}{\Delta x}+\mathrm{k} \frac{\Delta y}{2} \frac{T_{22}^{i}-T_{21}^{i}}{\Delta x}+\mathrm{k} \Delta x \frac{T_{16}^{i}-T_{21}^{i}}{\Delta y}=\rho \Delta x \frac{\Delta y}{2} \mathrm{C} \frac{T_{21}^{i+1}-T_{21}^{i}}{\Delta t}$

$T_{21}^{i+1}=\left(\left(h o \Delta x\left(T_{\infty}-T_{21}^{i}\right)+\mathrm{k} \frac{\Delta y}{2} \frac{T_{20}^{i}-T_{21}^{i}}{\Delta x}+\mathrm{k} \frac{\Delta y}{2} \frac{T_{22}^{i}-T_{21}^{i}}{\Delta x}+\mathrm{k} \Delta x \frac{T_{16}^{i}-T_{21}^{i}}{\Delta y}\right) \frac{2 \Delta t}{\rho(\Delta x \Delta y}\right)+T_{21}^{i}$

Node 22:

$h o \Delta x\left(T_{\infty}-T_{22}^{i}\right)+\mathrm{k} \frac{\Delta y}{2} \frac{T_{21}^{i}-T_{22}^{i}}{\Delta x}+\mathrm{k} \frac{\Delta y}{2} \frac{T_{23}^{i}-T_{22}^{i}}{\Delta x}+\mathrm{k} \Delta x \frac{T_{17}^{i}-T_{22}^{i}}{\Delta y}=\rho \Delta x \frac{\Delta y}{2} C \frac{T_{22}^{i+1}-T_{22}^{i}}{\Delta t}$

$T_{22}^{i+1}=\left(\left(h o \Delta x\left(T_{\infty}-T_{22}^{i}\right)+\mathrm{k} \frac{\Delta y}{2} \frac{T_{21}^{i}-T_{22}^{i}}{\Delta x}+\mathrm{k} \frac{\Delta y}{2} \frac{T_{23}^{i}-T_{22}^{i}}{\Delta x}+\mathrm{k} \Delta x \frac{T_{17}^{i}-T_{22}^{i}}{\Delta y}\right) \frac{2 \Delta t}{\rho(\Delta x \Delta y}\right)+T_{22}^{i}$

Node 23:

$h o \Delta x\left(T_{\infty}-T_{23}^{i}\right)+\mathrm{k} \frac{\Delta y}{2} \frac{T_{22}^{i}-T_{23}^{i}}{\Delta x}+\mathrm{k} \frac{\Delta y}{2} \frac{T_{24}^{i}-T_{23}^{i}}{\Delta x}+\mathrm{k} \Delta x \frac{T_{18}^{i}-T_{23}^{i}}{\Delta y}=\rho \Delta x \frac{\Delta y}{2} C \frac{T_{23}^{i+1}-T_{23}^{i}}{\Delta t}$

$T_{23}^{i+1}=\left(\left(h o \Delta x\left(T_{\infty}-T_{23}^{i}\right)+\mathrm{k} \frac{\Delta y}{2} \frac{T_{22}^{i}-T_{23}^{i}}{\Delta x}+\mathrm{k} \frac{\Delta y}{2} \frac{T_{24}^{i}-T_{23}^{i}}{\Delta x}+\mathrm{k} \Delta x \frac{T_{18}^{i}-T_{23}^{i}}{\Delta y}\right) \frac{2 \Delta t}{\rho(\Delta x \Delta y}\right)+T_{23}^{i}$

Node 24:

$h o \frac{\Delta x}{2}\left(T_{\infty}-T_{24}^{i}\right)+h i \frac{\Delta y}{2}\left(T_{h}-T_{24}^{i}\right)+\mathrm{k} \frac{\Delta y}{2} \frac{T_{33}^{i}-T_{24}^{i}}{\Delta x}+\mathrm{k} \frac{\Delta x}{2} \frac{T_{19}^{i}-T_{24}^{i}}{\Delta y}=\rho \frac{\Delta x}{2} \frac{\Delta y}{2} C \frac{T_{24}^{i+1}-T_{24}^{i}}{\Delta t}$

$T_{24}^{i+1}=\left(\left(h o \frac{\Delta x}{2}\left(T_{\infty}-T_{24}^{i}\right)+h i \frac{\Delta y}{2}\left(T_{h}-T_{24}^{i}\right)+\mathrm{k} \frac{\Delta y}{2} \frac{T_{23}^{i}-T_{24}^{i}}{\Delta x}+\mathrm{k} \frac{\Delta x}{2} \frac{T_{19}^{i}-T_{24}^{i}}{\Delta y}\right) \frac{4 \Delta t}{\rho(\Delta x \Delta y}\right)+T_{24}^{i}$ 


\section{Programming \& Solution}

With the help of a computer program we can solve the matrix created by finite difference equations for 24 nodes. We can calculate temperature distribution and stress distribution with respect to time.

Table 1Material Property and Boundary Conditions

\begin{tabular}{|l|l|l|l|}
\hline \multicolumn{3}{|l|}{$\begin{array}{l}\text { Material Properties and Boundary Conditions for Magnesia } \\
\text { Ramming Mass }\end{array}$} & Unit \\
\hline 1 & Internal Film Co-efficient hi & 200 & $\mathrm{~W} / \mathrm{m}^{2} \mathrm{~K}$ \\
\hline 2 & External Film Co-efficient ho & 40 & $\mathrm{~W} / \mathrm{m}^{2} \mathrm{~K}$ \\
\hline 3 & Atmosphere Film Co-efficient ha & 10 & $\mathrm{~W} / \mathrm{m}^{2} \mathrm{~K}$ \\
\hline 4 & Density & 3300 & $\mathrm{Kg} / \mathrm{m}^{3}$ \\
\hline 5 & Time Interval $\Delta \mathrm{t}$ & 10 & $\mathrm{Seconds}$ \\
\hline 6 & Thermal Conductivity k & 4 & $\mathrm{~W} / \mathrm{m} \mathrm{K}$ \\
\hline 7 & Temperature outside Furnace Wall & 303 & Kelvin \\
\hline 8 & Temperature inside Furnace Wall & 1773 & Kelvin \\
\hline 9 & Temperature of Air & 303 & Kelvin \\
\hline 10 & Specific Heat & 880 & $\mathrm{~J} / \mathrm{kg} \mathrm{K}$ \\
\hline 11 & Elasticity Constant & 180000 & $\mathrm{~N} / \mathrm{m}^{2}$ \\
\hline 12 & Thermal Expansion Co-efficient & 0.00000138 & $\mathrm{~m} / \mathrm{K}$ \\
\hline 13 & Ultimate Stress & 600 & $\mathrm{MPa}$ \\
\hline
\end{tabular}

\section{Computer Program}

\#include<iostream.h>

\#include<conio.h>

\#include<math.h>

\#include<complex.h>

\#include<fstream.h>

void main()

$\{$ clrscr;

ofstreammyfile;

myfile.open ("outputfdm.txt");

myfile<<"Writing this to a file. In";

float T[25][500];

float $S[25][500]$;

float hi,ho,ha,To,r,t,k,Ta,c,Th,E,Af,Sut,Se,m,N,L;

cout $<<$ "Enter in W/m2 K value of hi";

cin $>>$ hi;

cout $<<$ "Enter in W/m2 K value of ho";

cin $>>$ ho;

cout $<<$ "Enter in W/m2 K value of ha";

cin $>>$ ha;

cout<<"Enter in $\mathrm{Kg} / \mathrm{m} 3$ value of density";

cin $>r$;

cout $<<$ "Enter in seconds time interval delta t";

cin $>$ t;

cout $<<$ "Enter in W/m K value of thermal conductivity";

cin $>$ k;

cout $<<$ "Enter in Kelvin Temperature outside Furnace Wall";

cin $>$ Ta;

cout $<<$ "Enter in Kelvin Temperature inside Furnace Wall";

cin $>$ Th;

cout $<<$ "Enter in Kelvin Temperature of Air";

cin $>$ To;

cout $<<$ "Enter in J/Kg K value of Specific Heat";

cin $>$;

cout<<"Enter in N/m2 value of Elasticity Constant";

cin $>>E$;

cout $<<$ "Enter in $\mathrm{m} / \mathrm{K}$ Thermal Expansion Co-efficient";

cin $>$ Af;

cout $<<$ "Enter in MPA value of Ultimate Stress";

cin $>$ Sut: 
$\mathrm{T}[1][0]=300$

$\mathrm{T}[2][0]=300$;

$\mathrm{T}[3][0]=300 ;$

$\mathrm{T}[4][0]=300$;

$\mathrm{T}[5][0]=300 ;$

$\mathrm{T}[6][0]=300$;

$\mathrm{T}[7][0]=300$;

$\mathrm{T}[8][0]=300$;

$\mathrm{T}[9][0]=300$;

$\mathrm{T}[10][0]=300$;

$\mathrm{T}[11][0]=300$;

$\mathrm{T}[12][0]=300$;

$\mathrm{T}[13][0]=300$;

$\mathrm{T}[14][0]=300$;

$\mathrm{T}[15][0]=300$;

$\mathrm{T}[16][0]=300$;

$\mathrm{T}[17][0]=300$;

$\mathrm{T}[18][0]=300$;

$\mathrm{T}[19][0]=300$;

$\mathrm{T}[20][0]=300$;

$\mathrm{T}[21][0]=300$;

$\mathrm{T}[22][0]=300$;

$\mathrm{T}[23][0]=300$;

$\mathrm{T}[24][0]=300$;

for (inti $=0 ; \mathrm{i}<=270 ; \mathrm{i}++$ )

\{

$\mathrm{T}[1][\mathrm{i}+1]=\quad=\quad((0.5 * \mathrm{ha} * 0.06 *(\mathrm{To}-\mathrm{T}[1][\mathrm{i}])) \quad+(\mathrm{ho} * 0.3 *(\mathrm{Ta}-\mathrm{T}[1][\mathrm{i}]) / 2)+(0.5 * \mathrm{k} * 0.3 *(\mathrm{~T}[2][\mathrm{i}]-$ $\mathrm{T}[1][\mathrm{i}]) / 0.06)+(0.5 * \mathrm{k} * 0.06 *(\mathrm{~T}[4][\mathrm{i}]-\mathrm{T}[1][\mathrm{i}]) / 0.3)) *((4 * \mathrm{t}) /(\mathrm{r} * \mathrm{c} * 0.06 * 0.3)))+\mathrm{T}[1][\mathrm{i}] ;$

$\mathrm{T}[2][\mathrm{i}+1]=(((\mathrm{ha} * 0.06 *(\mathrm{To}-\mathrm{T}[2][\mathrm{i}]))+(0.5 * \mathrm{k} * 0.3 *(\mathrm{~T}[1][\mathrm{i}]-\mathrm{T}[2][\mathrm{i}]) / 0.06)+(0.5 * \mathrm{k} * 0.3 *(\mathrm{~T}[3][\mathrm{i}]-\mathrm{T}[2][\mathrm{i}]) / 0.06)$ $+(0.5 * \mathrm{k} * 0.06 *(\mathrm{~T}[5][\mathrm{i}]-\mathrm{T}[2][\mathrm{i}]) / 0.3)) *((2 * \mathrm{t}) /(\mathrm{r} * * * 0.06 * 0.3)))+\mathrm{T}[2][\mathrm{i}] ;$

$\mathrm{T}[3][\mathrm{i}+1]=(((\mathrm{ha} * 0.06 *(\mathrm{To}-\mathrm{T}[3][\mathrm{i}]) * 0.5) \quad+(\quad \mathrm{hi} * 0.3 *(\mathrm{Th}-\mathrm{T}[3][\mathrm{i}]) * 0.5 \quad) \quad+(0.5 * \mathrm{k} * 0.3 *(\mathrm{~T}[2][\mathrm{i}]-$ $\mathrm{T}[3][\mathrm{i}]) / 0.06)+(0.5 * \mathrm{k} * 0.06 *(\mathrm{~T}[6][\mathrm{i}]-\mathrm{T}[3][\mathrm{i}]) / 0.3)) *((4 * \mathrm{t}) /(\mathrm{r} * \mathrm{c} * 0.06 * 0.3)))+\mathrm{T}[3][\mathrm{i}] ;$

$\mathrm{T}[4][\mathrm{i}+1]=(((\mathrm{ho} * 0.3 *(\mathrm{Ta}-\mathrm{T}[4][\mathrm{i}]))+(\mathrm{k} * 0.3 *(\mathrm{~T}[5][\mathrm{i}]-\mathrm{T}[4][\mathrm{i}]) / 0.06)+(0.5 * \mathrm{k} * 0.06 *(\mathrm{~T}[1][\mathrm{i}]-\mathrm{T}[4][\mathrm{i}]) / 0.3)+$ $(0.5 * \mathrm{k} * 0.06 *(\mathrm{~T}[7][\mathrm{i}]-\mathrm{T}[4][\mathrm{i}]) / 0.3)) *((2 * \mathrm{t}) /(\mathrm{r} * \mathrm{c} * 0.06 * 0.3)))+\mathrm{T}[4][\mathrm{i}] ;$

$\mathrm{T}[5][\mathrm{i}+1]=(((\mathrm{k} * 0.3 *(\mathrm{~T}[4][\mathrm{i}]-\mathrm{T}[5][\mathrm{i}]) / 0.06)+(\mathrm{k} * 0.3 *(\mathrm{~T}[6][\mathrm{i}]-\mathrm{T}[5][\mathrm{i}]) / 0.06)+(\mathrm{k} * 0.06 *(\mathrm{~T}[8][\mathrm{i}]-\mathrm{T}[5][\mathrm{i}]) / 0.3))$ $*((\mathrm{t}) /(\mathrm{r} * \mathrm{c} * 0.06 * 0.3)))+\mathrm{T}[5][\mathrm{i}]$;

$\mathrm{T}[6][\mathrm{i}+1]=(((\mathrm{hi} * 0.3 *(\mathrm{Th}-\mathrm{T}[6][\mathrm{i}]))+(\mathrm{k} * 0.3 *(\mathrm{~T}[2][\mathrm{i}]-\mathrm{T}[6][\mathrm{i}]) / 0.06)+(0.5 * \mathrm{k} * 0.06 *(\mathrm{~T}[3][\mathrm{i}]-\mathrm{T}[6][\mathrm{i}]) / 0.3)+$ $(0.5 * \mathrm{k} * 0.06 *(\mathrm{~T}[9][\mathrm{i}]-\mathrm{T}[6][\mathrm{i}]) / 0.3)) *((2 * \mathrm{t}) /(\mathrm{r} * \mathrm{c} * 0.06 * 0.3)))+\mathrm{T}[6][\mathrm{i}] ;$

$\mathrm{T}[7][\mathrm{i}+1]=(((\mathrm{ho} * 0.3 *(\mathrm{Ta}-\mathrm{T}[7][\mathrm{i}]))+(\mathrm{k} * 0.3 *(\mathrm{~T}[8][\mathrm{i}]-\mathrm{T}[7][\mathrm{i}]) / 0.06)+(0.5 * \mathrm{k} * 0.06 *(\mathrm{~T}[4][\mathrm{i}]-\mathrm{T}[7][\mathrm{i}]) / 0.3)+$ $(0.5 * \mathrm{k} * 0.06 *(\mathrm{~T}[10][\mathrm{i}]-\mathrm{T}[7][\mathrm{i}]) / 0.3)) *((2 * \mathrm{t}) /(\mathrm{r} * \mathrm{c} * 0.06 * 0.3)))+\mathrm{T}[7][\mathrm{i}]$;

$\mathrm{T}[8][\mathrm{i}+1]=(((\mathrm{k} * 0.3 *(\mathrm{~T}[7][\mathrm{i}]-\mathrm{T}[8][\mathrm{i}]) / 0.06)+(\mathrm{k} * 0.3 *(\mathrm{~T}[9][\mathrm{i}]-\mathrm{T}[8][\mathrm{i}]) / 0.06)+(\mathrm{k} * 0.06 *(\mathrm{~T}[5][\mathrm{i}]-\mathrm{T}[8][\mathrm{i}]) / 0.3)+$ $(\mathrm{k} * 0.06 *(\mathrm{~T}[11][\mathrm{i}]-\mathrm{T}[8][\mathrm{i}]) / 0.3)) *((\mathrm{t}) /(\mathrm{r} * \mathrm{c} * 0.06 * 0.3)))+\mathrm{T}[8][\mathrm{i}] ;$

$\mathrm{T}[9][\mathrm{i}+1]=((\quad(\mathrm{hi} * 0.3 *(\mathrm{Th}-\mathrm{T}[9][\mathrm{i}]))+(\mathrm{k} * 0.3 *(\mathrm{~T}[8][\mathrm{i}]-\mathrm{T}[9][\mathrm{i}]) / 0.06)+\quad(0.5 * \mathrm{k} * 0.06 *(\mathrm{~T}[6][\mathrm{i}]-\mathrm{T}[9][\mathrm{i}]) / 0.3)+$ $(0.5 * \mathrm{k} * 0.06 *(\mathrm{~T}[12][\mathrm{i}]-\mathrm{T}[9][\mathrm{i}]) / 0.3)) *((2 * \mathrm{t}) /(\mathrm{r} * \mathrm{c} * 0.06 * 0.3)))+\mathrm{T}[9][\mathrm{i}] ;$

$\mathrm{T}[10][\mathrm{i}+1]=((\quad(\mathrm{ho} * 0.18 *(\mathrm{Ta}-\mathrm{T}[10][\mathrm{i}]))+(\mathrm{k} * 0.18 *(\mathrm{~T}[11][\mathrm{i}]-\mathrm{T}[10][\mathrm{i}]) / 0.06)+\quad(0.5 * \mathrm{k} * 0.06 *(\mathrm{~T}[7][\mathrm{i}]-$ $\mathrm{T}[10][\mathrm{i}]) / 0.18)+(0.5 * \mathrm{k} * 0.06 *(\mathrm{~T}[15][\mathrm{i}]-\mathrm{T}[10][\mathrm{i}]) / 0.18)) *((2 * \mathrm{t}) /(\mathrm{r} * \mathrm{c} * 0.06 * 0.18)))+\mathrm{T}[10][\mathrm{i}] ;$

$\mathrm{T}[11][\mathrm{i}+1]=((\quad(\mathrm{k} * 0.18 *(\mathrm{~T}[10][\mathrm{i}]-\mathrm{T}[11][\mathrm{i}]) / 0.06)+\quad(\mathrm{k} * 0.18 *(\mathrm{~T}[12][\mathrm{i}]-\mathrm{T}[11][\mathrm{i}]) / 0.06)+\quad(\mathrm{k} * 0.06 *(\mathrm{~T}[8][\mathrm{i}]-$ $\mathrm{T}[11][\mathrm{i}]) / 0.18)+(\mathrm{k} * 0.06 *(\mathrm{~T}[16][\mathrm{i}]-\mathrm{T}[11][\mathrm{i}]) / 0.18)) *((\mathrm{t}) /(\mathrm{r} * \mathrm{c} * 0.06 * 0.18)))+\mathrm{T}[11][\mathrm{i}] ;$

$\mathrm{T}[12][\mathrm{i}+1]=(((0.5 * \mathrm{hi} * 0.12 *(\mathrm{Th}-\mathrm{T}[12][\mathrm{i}]))+(0.5 * \mathrm{hi} * 0.18 *(\mathrm{Th}-\mathrm{T}[12][\mathrm{i}]))+(\mathrm{k} * 0.18 *(\mathrm{~T}[11][\mathrm{i}]-\mathrm{T}[12][\mathrm{i}]) / 0.12)+$ $(0.5 * \mathrm{k} * 0.18 *(\mathrm{~T}[13][\mathrm{i}]-\mathrm{T}[12][\mathrm{i}]) / 0.12)+(0.5 * \mathrm{k} * 0.12 *(\mathrm{~T}[9][\mathrm{i}]-\mathrm{T}[12][\mathrm{i}]) / 0.18)+(\mathrm{k} * 0.12 *(\mathrm{~T}[17][\mathrm{i}]-\mathrm{T}[12][\mathrm{i}]) / 0.18))$ $*((4 * \mathrm{t}) /(3 * \mathrm{r} * \mathrm{c} * 0.12 * 0.18)))+\mathrm{T}[12][\mathrm{i}]$

$\mathrm{T}[13][\mathrm{i}+1]=((\quad(\mathrm{hi} * 0.18 *(\mathrm{Th}-\mathrm{T}[13][\mathrm{i}]))+(0.5 * \mathrm{k} * 0.18 *(\mathrm{~T}[12][\mathrm{i}]-\mathrm{T}[13][\mathrm{i}]) / 0.18)+\quad(0.5 * \mathrm{k} * 0.18 *(\mathrm{~T}[14][\mathrm{i}]-$ $\mathrm{T}[13][\mathrm{i}]) / 0.18)+(\mathrm{k} * 0.18 *(\mathrm{~T}[18][\mathrm{i}]-\mathrm{T}[13][\mathrm{i}]) / 0.18)) *((2 * \mathrm{t}) /(\mathrm{r} * \mathrm{c} * 0.18 * 0.18)))+\mathrm{T}[13][\mathrm{i}]$;

$\mathrm{T}[14][\mathrm{i}+1]=((\quad(0.5 * \mathrm{hi} * 0.18 *(\mathrm{Th}-\mathrm{T}[14][\mathrm{i}]))+(0.5 * \mathrm{hi} * 0.18 *(\mathrm{Th}-\mathrm{T}[14][\mathrm{i}]))+(0.5 * \mathrm{k} * 0.18 *(\mathrm{~T}[13][\mathrm{i}]-$ $\mathrm{T}[14][\mathrm{i}]) / 0.18)+(0.5 * \mathrm{k} * 0.18 *(\mathrm{~T}[19][\mathrm{i}]-\mathrm{T}[14][\mathrm{i}]) / 0.18)) *((4 * \mathrm{t}) /(\mathrm{r} * \mathrm{c} * 0.18 * 0.18)))+\mathrm{T}[14][\mathrm{i}] ;$

$\mathrm{T}[15][\mathrm{i}+1]=((\quad(\mathrm{ho} * 0.06 *(\mathrm{Ta}-\mathrm{T}[15][\mathrm{i}]))+(\mathrm{k} * 0.06 *(\mathrm{~T}[16][\mathrm{i}]-\mathrm{T}[15][\mathrm{i}]) / 0.06)+(0.5 * \mathrm{k} * 0.06 *(\mathrm{~T}[10][\mathrm{i}]-$ $\mathrm{T}[15][\mathrm{i}]) / 0.06)+(0.5 * \mathrm{k} * 0.06 *(\mathrm{~T}[20][\mathrm{i}]-\mathrm{T}[15][\mathrm{i}]) / 0.06)) *((2 * \mathrm{t}) /(\mathrm{r} * \mathrm{c} * 0.06 * 0.06)))+\mathrm{T}[15][\mathrm{i}]$; 
$\mathrm{T}[16][\mathrm{i}+1]=\left(\left(\quad\left(\mathrm{k}^{*} 0.06 *(\mathrm{~T}[15][\mathrm{i}]-\mathrm{T}[16][\mathrm{i}]) / 0.06\right)+\left(\mathrm{k}^{*} 0.06 *(\mathrm{~T}[17][\mathrm{i}]-\mathrm{T}[16][\mathrm{i}]) / 0.06 \quad\right)+\left(\mathrm{k}^{*} 0.06 *(\mathrm{~T}[11][\mathrm{i}]-\right.\right.\right.$ $\mathrm{T}[16][\mathrm{i}]) / 0.06)+(\mathrm{k} * 0.06 *(\mathrm{~T}[21][\mathrm{i}]-\mathrm{T}[16][\mathrm{i}]) / 0.06)) *(\mathrm{t}) /(\mathrm{r} * \mathrm{c} * 0.06 * 0.06)))+\mathrm{T}[16][\mathrm{i}] ;$

$\mathrm{T}[17][\mathrm{i}+1]=((\quad(\mathrm{k} * 0.06 *(\mathrm{~T}[16][\mathrm{i}]-\mathrm{T}[17][\mathrm{i}]) / 0.12)+(\mathrm{k} * 0.06 *(\mathrm{~T}[18][\mathrm{i}]-\mathrm{T}[17][\mathrm{i}]) / 0.12)+(\mathrm{k} * 0.12 *(\mathrm{~T}[12][\mathrm{i}]-$ $\mathrm{T}[17][\mathrm{i}]) / 0.06)+(\mathrm{k} * 0.12 *(\mathrm{~T}[22][\mathrm{i}]-\mathrm{T}[17][\mathrm{i}]) / 0.06)) *(\mathrm{t}) /(\mathrm{r} * \mathrm{c} * 0.12 * 0.06)))+\mathrm{T}[17][\mathrm{i}] ;$

$\mathrm{T}[18][\mathrm{i}+1]=\left(\left(\quad\left(\mathrm{k}^{*} 0.06 *(\mathrm{~T}[17][\mathrm{i}]-\mathrm{T}[18][\mathrm{i}]) / 0.18\right)+\left(\mathrm{k}^{*} 0.06 *(\mathrm{~T}[19][\mathrm{i}]-\mathrm{T}[18][\mathrm{i}]) / 0.18\right)+\left(\mathrm{k}^{*} 0.18 *(\mathrm{~T}[13][\mathrm{i}]-\right.\right.\right.$ $\left.\mathrm{T}[18][\mathrm{i}]) / 0.06)+(\mathrm{k} * 0.18 *(\mathrm{~T}[23][\mathrm{i}]-\mathrm{T}[18][\mathrm{i}]) / 0.06)) *\left((\mathrm{t}) /\left(\mathrm{r} * \mathrm{c}^{*} 0.18 * 0.06\right)\right)\right)+\mathrm{T}[18][\mathrm{i}] ;$

$\mathrm{T}[19][\mathrm{i}+1]=\left(\left(\quad\left(\mathrm{hi}^{*} 0.06 *(\mathrm{Th}-\mathrm{T}[19][\mathrm{i}])\right)+(\mathrm{k} * 0.06 *(\mathrm{~T}[18][\mathrm{i}]-\mathrm{T}[19][\mathrm{i}]) / 0.18)+\quad\left(0.5 * \mathrm{k}^{*} 0.18 *(\mathrm{~T}[14][\mathrm{i}]-\right.\right.\right.$ $\mathrm{T}[19][\mathrm{i}]) / 0.06)+(0.5 * \mathrm{k} * 0.18 *(\mathrm{~T}[24][\mathrm{i}]-\mathrm{T}[19][\mathrm{i}]) / 0.06)) *((2 * \mathrm{t}) /(\mathrm{r} * \mathrm{c} * 0.18 * 0.06)))+\mathrm{T}[19][\mathrm{i}]$;

$\mathrm{T}[20][\mathrm{i}+1]=\left(\left(\quad\left(0.5 * \mathrm{ho}^{*} 0.06 *(\mathrm{Ta}-\mathrm{T}[20][\mathrm{i}])\right)+(\quad 0.5 * \mathrm{ho} * 0.06 *(\mathrm{Ta}-\mathrm{T}[20][\mathrm{i}]))+(0.5 * \mathrm{k} * 0.06 *(\mathrm{~T}[21][\mathrm{i}]-\right.\right.$ $\mathrm{T}[20][\mathrm{i}]) / 0.06)+(0.5 * \mathrm{k} * 0.06 *(\mathrm{~T}[15][\mathrm{i}]-\mathrm{T}[20][\mathrm{i}]) / 0.06)) *((4 * \mathrm{t}) /(\mathrm{r} * \mathrm{c} * 0.06 * 0.06)))+\mathrm{T}[20][\mathrm{i}] ;$

$\mathrm{T}[21][\mathrm{i}+1]=((\quad(0.5 * \mathrm{ho} * 0.06 *(\mathrm{Ta}-\mathrm{T}[21][\mathrm{i}]))+(0.5 * \mathrm{k} * 0.06 *(\mathrm{~T}[20][\mathrm{i}]-\mathrm{T}[21][\mathrm{i}]) / 0.06)+(0.5 * \mathrm{k} * 0.06 *(\mathrm{~T}[22][\mathrm{i}]-$ $\mathrm{T}[21][\mathrm{i}]) / 0.06)+(\mathrm{k} * 0.06 *(\mathrm{~T}[16][\mathrm{i}]-\mathrm{T}[21][\mathrm{i}]) / 0.06)) *((2 * \mathrm{t}) /(\mathrm{r} * \mathrm{c} * 0.06 * 0.06)))+\mathrm{T}[21][\mathrm{i}]$;

$\mathrm{T}[22][\mathrm{i}+1]=((\quad(\mathrm{ho} * 0.12 *(\mathrm{Ta}-\mathrm{T}[22][\mathrm{i}]))+(0.5 * \mathrm{k} * 0.06 *(\mathrm{~T}[21][\mathrm{i}]-\mathrm{T}[22][\mathrm{i}]) / 0.12)+(\quad 0.5 * \mathrm{k} * 0.06 *(\mathrm{~T}[23][\mathrm{i}]-$ $\mathrm{T}[22][\mathrm{i}]) / 0.12)+(\mathrm{k} * 0.12 *(\mathrm{~T}[17][\mathrm{i}]-\mathrm{T}[22][\mathrm{i}]) / 0.06)) *((2 * \mathrm{t}) /(\mathrm{r} * \mathrm{c} * 0.12 * 0.06)))+\mathrm{T}[22][\mathrm{i}]$;

$\mathrm{T}[23][\mathrm{i}+1]=((\quad(\mathrm{ho} * 0.18 *(\mathrm{Ta}-\mathrm{T}[23][\mathrm{i}]))+(0.5 * \mathrm{k} * 0.06 *(\mathrm{~T}[22][\mathrm{i}]-\mathrm{T}[23][\mathrm{i}]) / 0.18)+(\quad 0.5 * \mathrm{k} * 0.06 *(\mathrm{~T}[24][\mathrm{i}]-$ $\mathrm{T}[23][\mathrm{i}]) / 0.18)+(\mathrm{k} * 0.18 *(\mathrm{~T}[18][\mathrm{i}]-\mathrm{T}[23][\mathrm{i}]) / 0.06)) *((2 * \mathrm{t}) /(\mathrm{r} * \mathrm{c} * 0.18 * 0.06)))+\mathrm{T}[23][\mathrm{i}]$;

$\mathrm{T}[24][\mathrm{i}+1]=((\quad(0.5 * \mathrm{ho} * 0.18 *(\mathrm{Ta}-\mathrm{T}[24][\mathrm{i}]))+(\quad 0.5 * \mathrm{hi} * 0.06 *(\mathrm{Th}-\mathrm{T}[24][\mathrm{i}]))+(0.5 * \mathrm{k} * 0.06 *(\mathrm{~T}[23][\mathrm{i}]-$ $\mathrm{T}[24][\mathrm{i}]) / 0.18)+(0.5 * \mathrm{k} * 0.18 *(\mathrm{~T}[19][\mathrm{i}]-\mathrm{T}[24][\mathrm{i}]) / 0.06)) *((4 * \mathrm{t}) /(\mathrm{r} * \mathrm{c} * 0.18 * 0.06)))+\mathrm{T}[24][\mathrm{i}] ;$

$\mathrm{S}[14][\mathrm{i}]=\mathrm{E} * \mathrm{Af} * \mathrm{~T}[14][\mathrm{i}]$;

\}

for $(\mathrm{i}=271 ; \mathrm{i}<=360 ; \mathrm{i}++)$

\{

hi=ha;

$\mathrm{Th}=\mathrm{Ta}$;

$\mathrm{T}[1][\mathrm{i}+1]=\quad(((0.5 * \mathrm{ha} * 0.06 *(\mathrm{To}-\mathrm{T}[1][\mathrm{i}])) \quad+(\mathrm{ho} * 0.3 *(\mathrm{Ta}-\mathrm{T}[1][\mathrm{i}]) / 2)+(0.5 * \mathrm{k} * 0.3 *(\mathrm{~T}[2][\mathrm{i}]-$

$\mathrm{T}[1][\mathrm{i}]) / 0.06)+(0.5 * \mathrm{k} * 0.06 *(\mathrm{~T}[4][\mathrm{i}]-\mathrm{T}[1][\mathrm{i}]) / 0.3)) *((4 * \mathrm{t}) /(\mathrm{r} * \mathrm{c} * 0.06 * 0.3)))+\mathrm{T}[1][\mathrm{i}] ;$

$\mathrm{T}[2][\mathrm{i}+1]=(((\mathrm{ha} * 0.06 *(\mathrm{To}-\mathrm{T}[2][\mathrm{i}]))+(0.5 * \mathrm{k} * 0.3 *(\mathrm{~T}[1][\mathrm{i}]-\mathrm{T}[2][\mathrm{i}]) / 0.06)+(0.5 * \mathrm{k} * 0.3 *(\mathrm{~T}[3][\mathrm{i}]-\mathrm{T}[2][\mathrm{i}]) / 0.06)$ $+(0.5 * \mathrm{k} * 0.06 *(\mathrm{~T}[5][\mathrm{i}]-\mathrm{T}[2][\mathrm{i}]) / 0.3)) *((2 * \mathrm{t}) /(\mathrm{r} * \mathrm{c} * 0.06 * 0.3)))+\mathrm{T}[2][\mathrm{i}] ;$

$\mathrm{T}[3][\mathrm{i}+1]=(((\mathrm{ha} * 0.06 *(\mathrm{To}-\mathrm{T}[3][\mathrm{i}]) * 0.5) \quad+($ hi*0.3*(Th-T[3][i] $) * 0.5) \quad+(0.5 * \mathrm{k} * 0.3 *(\mathrm{~T}[2][\mathrm{i}]-$ $\mathrm{T}[3][\mathrm{i}]) / 0.06)+(0.5 * \mathrm{k} * 0.06 *(\mathrm{~T}[6][\mathrm{i}]-\mathrm{T}[3][\mathrm{i}]) / 0.3)) *((4 * \mathrm{t}) /(\mathrm{r} * \mathrm{c} * 0.06 * 0.3)))+\mathrm{T}[3][\mathrm{i}] ;$

$\mathrm{T}[4][\mathrm{i}+1]=((($ ho $* 0.3 *(\mathrm{Ta}-\mathrm{T}[4][\mathrm{i}]))+(\mathrm{k} * 0.3 *(\mathrm{~T}[5][\mathrm{i}]-\mathrm{T}[4][\mathrm{i}]) / 0.06)+(0.5 * \mathrm{k} * 0.06 *(\mathrm{~T}[1][\mathrm{i}]-\mathrm{T}[4][\mathrm{i}]) / 0.3)+$ $(0.5 * \mathrm{k} * 0.06 *(\mathrm{~T}[7][\mathrm{i}]-\mathrm{T}[4][\mathrm{i}]) / 0.3)) *((2 * \mathrm{t}) /(\mathrm{r} * \mathrm{c} * 0.06 * 0.3)))+\mathrm{T}[4][\mathrm{i}]$;

$\mathrm{T}[5][\mathrm{i}+1]=(((\mathrm{k} * 0.3 *(\mathrm{~T}[4][\mathrm{i}]-\mathrm{T}[5][\mathrm{i}]) / 0.06)+(\mathrm{k} * 0.3 *(\mathrm{~T}[6][\mathrm{i}]-\mathrm{T}[5][\mathrm{i}]) / 0.06)+(\mathrm{k} * 0.06 *(\mathrm{~T}[8][\mathrm{i}]-\mathrm{T}[5][\mathrm{i}]) / 0.3))$ $*((\mathrm{t}) /(\mathrm{r} * \mathrm{c} * 0.06 * 0.3)))+\mathrm{T}[5][\mathrm{i}]$;

$\mathrm{T}[6][\mathrm{i}+1]=(((\mathrm{hi} * 0.3 *(\mathrm{Th}-\mathrm{T}[6][\mathrm{i}]))+(\mathrm{k} * 0.3 *(\mathrm{~T}[2][\mathrm{i}]-\mathrm{T}[6][\mathrm{i}]) / 0.06)+(0.5 * \mathrm{k} * 0.06 *(\mathrm{~T}[3][\mathrm{i}]-\mathrm{T}[6][\mathrm{i}]) / 0.3)+$ $(0.5 * \mathrm{k} * 0.06 *(\mathrm{~T}[9][\mathrm{i}]-\mathrm{T}[6][\mathrm{i}]) / 0.3)) *((2 * \mathrm{t}) /(\mathrm{r} * \mathrm{c} * 0.06 * 0.3)))+\mathrm{T}[6][\mathrm{i}]$;

$\mathrm{T}[7][\mathrm{i}+1]=(((\mathrm{ho} * 0.3 *(\mathrm{Ta}-\mathrm{T}[7][\mathrm{i}]))+(\mathrm{k} * 0.3 *(\mathrm{~T}[8][\mathrm{i}]-\mathrm{T}[7][\mathrm{i}]) / 0.06)+(0.5 * \mathrm{k} * 0.06 *(\mathrm{~T}[4][\mathrm{i}]-\mathrm{T}[7][\mathrm{i}]) / 0.3)+$ $(0.5 * \mathrm{k} * 0.06 *(\mathrm{~T}[10][\mathrm{i}]-\mathrm{T}[7][\mathrm{i}]) / 0.3)) *((2 * \mathrm{t}) /(\mathrm{r} * \mathrm{c} * 0.06 * 0.3)))+\mathrm{T}[7][\mathrm{i}] ;$

$\mathrm{T}[8][\mathrm{i}+1]=(((\mathrm{k} * 0.3 *(\mathrm{~T}[7][\mathrm{i}]-\mathrm{T}[8][\mathrm{i}]) / 0.06)+(\mathrm{k} * 0.3 *(\mathrm{~T}[9][\mathrm{i}]-\mathrm{T}[8][\mathrm{i}]) / 0.06)+(\mathrm{k} * 0.06 *(\mathrm{~T}[5][\mathrm{i}]-\mathrm{T}[8][\mathrm{i}]) / 0.3)+$ $(\mathrm{k} * 0.06 *(\mathrm{~T}[11][\mathrm{i}]-\mathrm{T}[8][\mathrm{i}]) /(0.3)) *((\mathrm{t}) /(\mathrm{r} * \mathrm{c} * 0.06 * 0.3)))+\mathrm{T}[8][\mathrm{i}] ;$

$\mathrm{T}[9][\mathrm{i}+1]=\left(\left((\mathrm{hi} * 0.3 *(\mathrm{Th}-\mathrm{T}[9][\mathrm{i}]))+(\mathrm{k} * 0.3 *(\mathrm{~T}[8][\mathrm{i}]-\mathrm{T}[9][\mathrm{i}]) / 0.06)+\quad\left(0.5 * \mathrm{k}^{*} 0.06 *(\mathrm{~T}[6][\mathrm{i}]-\mathrm{T}[9][\mathrm{i}]) / 0.3\right)+\right.\right.$ $\left.(0.5 * \mathrm{k} * 0.06 *(\mathrm{~T}[12][\mathrm{i}]-\mathrm{T}[9][\mathrm{i}]) / 0.3)) *\left((2 * \mathrm{t}) /\left({ }^{*}{ }^{*} * 0.06 * 0.3\right)\right)\right)+\mathrm{T}[9][\mathrm{i}]$;

$\mathrm{T}[10][\mathrm{i}+1]=((\quad($ ho $* 0.18 *(\mathrm{Ta}-\mathrm{T}[10][\mathrm{i}]))+(\mathrm{k} * 0.18 *(\mathrm{~T}[11][\mathrm{i}]-\mathrm{T}[10][\mathrm{i}]) / 0.06)+\quad(0.5 * \mathrm{k} * 0.06 *(\mathrm{~T}[7][\mathrm{i}]-$ $\mathrm{T}[10][\mathrm{i}]) / 0.18)+(0.5 * \mathrm{k} * 0.06 *(\mathrm{~T}[15][\mathrm{i}]-\mathrm{T}[10][\mathrm{i}]) / 0.18)) *((2 * \mathrm{t}) /(\mathrm{r} * \mathrm{c} * 0.06 * 0.18)))+\mathrm{T}[10][\mathrm{i}] ;$

$\mathrm{T}[11][\mathrm{i}+1]=((\quad(\mathrm{k} * 0.18 *(\mathrm{~T}[10][\mathrm{i}]-\mathrm{T}[11][\mathrm{i}]) / 0.06)+\quad(\mathrm{k} * 0.18 *(\mathrm{~T}[12][\mathrm{i}]-\mathrm{T}[11][\mathrm{i}]) / 0.06)+\quad(\mathrm{k} * 0.06 *(\mathrm{~T}[8][\mathrm{i}]-$ $\mathrm{T}[11][\mathrm{i}]) / 0.18)+(\mathrm{k} * 0.06 *(\mathrm{~T}[16][\mathrm{i}]-\mathrm{T}[11][\mathrm{i}]) / 0.18)) *((\mathrm{t}) /(\mathrm{r} * \mathrm{c} * 0.06 * 0.18)))+\mathrm{T}[11][\mathrm{i}] ;$

$\mathrm{T}[12][\mathrm{i}+1]=(((0.5 * \mathrm{hi} * 0.12 *(\mathrm{Th}-\mathrm{T}[12][\mathrm{i}]))+(0.5 * \mathrm{hi} * 0.18 *(\mathrm{Th}-\mathrm{T}[12][\mathrm{i}]))+(\mathrm{k} * 0.18 *(\mathrm{~T}[11][\mathrm{i}]-\mathrm{T}[12][\mathrm{i}]) / 0.12)+$ $\left.\left(0.5 * \mathrm{k}^{*} 0.18 *(\mathrm{~T}[13][\mathrm{i}]-\mathrm{T}[12][\mathrm{i}]) / 0.12\right)+\left(0.5 * \mathrm{k}^{*} 0.12 *(\mathrm{~T}[9][\mathrm{i}]-\mathrm{T}[12][\mathrm{i}]) / 0.18\right)+(\mathrm{k} * 0.12 *(\mathrm{~T}[17][\mathrm{i}]-\mathrm{T}[12][\mathrm{i}]) / 0.18)\right)$ $*((4 * \mathrm{t}) /(3 * \mathrm{r} * \mathrm{c} * 0.12 * 0.18)))+\mathrm{T}[12][\mathrm{i}]$

$\mathrm{T}[13][\mathrm{i}+1]=((\quad(\mathrm{hi} * 0.18 *(\mathrm{Th}-\mathrm{T}[13][\mathrm{i}]))+(0.5 * \mathrm{k} * 0.18 *(\mathrm{~T}[12][\mathrm{i}]-\mathrm{T}[13][\mathrm{i}]) / 0.18)+\quad(0.5 * \mathrm{k} * 0.18 *(\mathrm{~T}[14][\mathrm{i}]-$ $\mathrm{T}[13][\mathrm{i}]) / 0.18)+(\mathrm{k} * 0.18 *(\mathrm{~T}[18][\mathrm{i}]-\mathrm{T}[13][\mathrm{i}]) / 0.18)) *((2 * \mathrm{t}) /(\mathrm{r} * \mathrm{c} * 0.18 * 0.18)))+\mathrm{T}[13][\mathrm{i}]$

$\mathrm{T}[14][\mathrm{i}+1]=((\quad(0.5 * \mathrm{hi} * 0.18 *(\mathrm{Th}-\mathrm{T}[14][\mathrm{i}]))+(0.5 * \mathrm{hi} * 0.18 *(\mathrm{Th}-\mathrm{T}[14][\mathrm{i}]))+(0.5 * \mathrm{k} * 0.18 *(\mathrm{~T}[13][\mathrm{i}]-$ $\mathrm{T}[14][\mathrm{i}]) / 0.18)+(0.5 * \mathrm{k} * 0.18 *(\mathrm{~T}[19][\mathrm{i}]-\mathrm{T}[14][\mathrm{i}]) / 0.18)) *((4 * \mathrm{t}) /(\mathrm{r} * \mathrm{c} * 0.18 * 0.18)))+\mathrm{T}[14][\mathrm{i}]$;

$\mathrm{T}[15][\mathrm{i}+1]=((\quad(\mathrm{ho} * 0.06 *(\mathrm{Ta}-\mathrm{T}[15][\mathrm{i}]))+(\mathrm{k} * 0.06 *(\mathrm{~T}[16][\mathrm{i}]-\mathrm{T}[15][\mathrm{i}]) / 0.06)+\quad(0.5 * \mathrm{k} * 0.06 *(\mathrm{~T}[10][\mathrm{i}]-$ $\mathrm{T}[15][\mathrm{i}]) / 0.06)+(0.5 * \mathrm{k} * 0.06 *(\mathrm{~T}[20][\mathrm{i}]-\mathrm{T}[15][\mathrm{i}]) / 0.06)) *((2 * \mathrm{t}) /(\mathrm{r} * \mathrm{c} * 0.06 * 0.06)))+\mathrm{T}[15][\mathrm{i}]$;

$\mathrm{T}[16][\mathrm{i}+1]=\left(\left(\quad \mathrm{k}^{*} 0.06 *(\mathrm{~T}[15][\mathrm{i}]-\mathrm{T}[16][\mathrm{i}]) / 0.06\right)+\left(\mathrm{k}^{*} 0.06 *(\mathrm{~T}[17][\mathrm{i}]-\mathrm{T}[16][\mathrm{i}]) / 0.06\right)+\left(\mathrm{k}^{*} 0.06 *(\mathrm{~T}[11][\mathrm{i}]-\right.\right.$ $\mathrm{T}[16][\mathrm{i}]) / 0.06)+(\mathrm{k} * 0.06 *(\mathrm{~T}[21][\mathrm{i}]-\mathrm{T}[16][\mathrm{i}]) / 0.06)) *((\mathrm{t}) /(\mathrm{r} * \mathrm{c} * 0.06 * 0.06)))+\mathrm{T}[16][\mathrm{i}]$; 
$\mathrm{T}[17][\mathrm{i}+1]=\left(\left(\quad\left(\mathrm{k}^{*} 0.06 *(\mathrm{~T}[16][\mathrm{i}]-\mathrm{T}[17][\mathrm{i}]) / 0.12\right)+(\mathrm{k} * 0.06 *(\mathrm{~T}[18][\mathrm{i}]-\mathrm{T}[17][\mathrm{i}]) / 0.12 \quad)+(\mathrm{k} * 0.12 *(\mathrm{~T}[12][\mathrm{i}]-\right.\right.$ $\mathrm{T}[17][\mathrm{i}]) / 0.06)+(\mathrm{k} * 0.12 *(\mathrm{~T}[22][\mathrm{i}]-\mathrm{T}[17][\mathrm{i}]) / 0.06)) *(\mathrm{t}) /(\mathrm{r} * \mathrm{c} * 0.12 * 0.06)))+\mathrm{T}[17][\mathrm{i}] ;$

$\mathrm{T}[18][\mathrm{i}+1]=\left(\left(\quad\left(\mathrm{k}^{*} 0.06 *(\mathrm{~T}[17][\mathrm{i}]-\mathrm{T}[18][\mathrm{i}]) / 0.18\right)+(\mathrm{k} * 0.06 *(\mathrm{~T}[19][\mathrm{i}]-\mathrm{T}[18][\mathrm{i}]) / 0.18)+(\mathrm{k} * 0.18 *(\mathrm{~T}[13][\mathrm{i}]-\right.\right.$ $\left.\mathrm{T}[18][\mathrm{i}]) / 0.06)+(\mathrm{k} * 0.18 *(\mathrm{~T}[23][\mathrm{i}]-\mathrm{T}[18][\mathrm{i}]) / 0.06)) *\left((\mathrm{t}) /\left(\mathrm{r} * \mathrm{c}^{*} 0.18 * 0.06\right)\right)\right)+\mathrm{T}[18][\mathrm{i}] ;$

$\mathrm{T}[19][\mathrm{i}+1]=\left(\left(\quad\left(\mathrm{hi}^{*} 0.06 *(\mathrm{Th}-\mathrm{T}[19][\mathrm{i}])\right)+(\mathrm{k} * 0.06 *(\mathrm{~T}[18][\mathrm{i}]-\mathrm{T}[19][\mathrm{i}]) / 0.18)+\quad\left(0.5 * \mathrm{k} * 0.18^{*}(\mathrm{~T}[14][\mathrm{i}]-\right.\right.\right.$ $\mathrm{T}[19][\mathrm{i}]) / 0.06)+(0.5 * \mathrm{k} * 0.18 *(\mathrm{~T}[24][\mathrm{i}]-\mathrm{T}[19][\mathrm{i}]) / 0.06)) *((2 * \mathrm{t}) /(\mathrm{r} * \mathrm{c} * 0.18 * 0.06)))+\mathrm{T}[19][\mathrm{i}]$;

$\mathrm{T}[20][\mathrm{i}+1]=((\quad(0.5 * \mathrm{ho} * 0.06 *(\mathrm{Ta}-\mathrm{T}[20][\mathrm{i}]))+(\quad 0.5 * \mathrm{ho} * 0.06 *(\mathrm{Ta}-\mathrm{T}[20][\mathrm{i}]))+(0.5 * \mathrm{k} * 0.06 *(\mathrm{~T}[21][\mathrm{i}]-$ $\mathrm{T}[20][\mathrm{i}]) / 0.06)+(0.5 * \mathrm{k} * 0.06 *(\mathrm{~T}[15][\mathrm{i}]-\mathrm{T}[20][\mathrm{i}]) / 0.06)) *((4 * \mathrm{t}) /(\mathrm{r} * \mathrm{c} * 0.06 * 0.06)))+\mathrm{T}[20][\mathrm{i}] ;$

$\mathrm{T}[21][\mathrm{i}+1]=((\quad(0.5 * \mathrm{ho} * 0.06 *(\mathrm{Ta}-\mathrm{T}[21][\mathrm{i}]))+(0.5 * \mathrm{k} * 0.06 *(\mathrm{~T}[20][\mathrm{i}]-\mathrm{T}[21][\mathrm{i}]) / 0.06)+(0.5 * \mathrm{k} * 0.06 *(\mathrm{~T}[22][\mathrm{i}]-$ $\mathrm{T}[21][\mathrm{i}]) / 0.06)+(\mathrm{k} * 0.06 *(\mathrm{~T}[16][\mathrm{i}]-\mathrm{T}[21][\mathrm{i}]) / 0.06)) *((2 * \mathrm{t}) /(\mathrm{r} * \mathrm{c} * 0.06 * 0.06)))+\mathrm{T}[21][\mathrm{i}]$;

$\mathrm{T}[22][\mathrm{i}+1]=((\quad(\mathrm{ho} * 0.12 *(\mathrm{Ta}-\mathrm{T}[22][\mathrm{i}]))+(0.5 * \mathrm{k} * 0.06 *(\mathrm{~T}[21][\mathrm{i}]-\mathrm{T}[22][\mathrm{i}]) / 0.12)+(\quad 0.5 * \mathrm{k} * 0.06 *(\mathrm{~T}[23][\mathrm{i}]-$ $\mathrm{T}[22][\mathrm{i}]) / 0.12)+(\mathrm{k} * 0.12 *(\mathrm{~T}[17][\mathrm{i}]-\mathrm{T}[22][\mathrm{i}]) / 0.06)) *((2 * \mathrm{t}) /(\mathrm{r} * \mathrm{c} * 0.12 * 0.06)))+\mathrm{T}[22][\mathrm{i}]$;

$\mathrm{T}[23][\mathrm{i}+1]=((\quad(\mathrm{ho} * 0.18 *(\mathrm{Ta}-\mathrm{T}[23][\mathrm{i}]))+(0.5 * \mathrm{k} * 0.06 *(\mathrm{~T}[22][\mathrm{i}]-\mathrm{T}[23][\mathrm{i}]) / 0.18)+(\quad 0.5 * \mathrm{k} * 0.06 *(\mathrm{~T}[24][\mathrm{i}]-$ $\mathrm{T}[23][\mathrm{i}]) / 0.18)+(\mathrm{k} * 0.18 *(\mathrm{~T}[18][\mathrm{i}]-\mathrm{T}[23][\mathrm{i}]) / 0.06)) *((2 * \mathrm{t}) /(\mathrm{r} * \mathrm{c} * 0.18 * 0.06)))+\mathrm{T}[23][\mathrm{i}]$;

$\mathrm{T}[24][\mathrm{i}+1]=((\quad(0.5 * \mathrm{ho} * 0.18 *(\mathrm{Ta}-\mathrm{T}[24][\mathrm{i}]))+(\quad 0.5 * \mathrm{hi} * 0.06 *(\mathrm{Th}-\mathrm{T}[24][\mathrm{i}]))+(0.5 * \mathrm{k} * 0.06 *(\mathrm{~T}[23][\mathrm{i}]-$ $\mathrm{T}[24][\mathrm{i}]) / 0.18)+(0.5 * \mathrm{k} * 0.18 *(\mathrm{~T}[19][\mathrm{i}]-\mathrm{T}[24][\mathrm{i}]) / 0.06)) *((4 * \mathrm{t}) /(\mathrm{r} * \mathrm{c} * 0.18 * 0.06)))+\mathrm{T}[24][\mathrm{i}] ;$

$\mathrm{S}[14][\mathrm{i}]=\mathrm{E}^{*} \mathrm{Af} * \mathrm{~T}[14][\mathrm{i}]$;

\}

for $(\mathrm{i}=0 ; \mathrm{i}<=360 ; \mathrm{i}=\mathrm{i}+6)$

\{

myfile<<"MAXIMUM TEMPERATURE AFTER "<<i/6<<" MINUTES"<<T[14][i] $<<"$ KELVIN"<<endl; myfile $<<"$ MAXIMUM STRESS AFTER "<<i/6<<" MINUTES "<<S[14][i] $<<"$ MPa" $<<$ endl;

for $(\mathrm{i}=0 ; \mathrm{i}<=300 ; \mathrm{i}++)$

\{

if $(i==270)$

\{

myfile $<<"$ S" $<<$ S[14][i];

$\mathrm{Se}=0.15 * \mathrm{Sut}$

$\mathrm{m}=(\mathrm{Sut}-\mathrm{Se}) / 7$;

$\mathrm{N}=7-((\mathrm{S}[14][\mathrm{i}]-\mathrm{Se}) / \mathrm{m})$;

$\mathrm{L}=\operatorname{pow}(10, \mathrm{~N})$;

myfile $<<" N "<<\mathrm{N}$;

myfile $<<" L I F E ~ C Y C L E "<<$;

myfile.close();

\}

\}

$\operatorname{getch}() ; \quad\}$

\section{Results and Discussion}

We can see from the Fig. 2 that maximum temperature is increasing from atmospheric temperature 300 $\mathrm{K}$ and reaches to maximum temperature $1667 \mathrm{~K}$ in 45 minutes and then starts reducing and reaches to $831 \mathrm{~K}$ in next 15 minutes. It again starts increasing and reaches to maximum $1667 \mathrm{~K}$ after 105 minutes and again starts reducing. There are 10 similar temperature cycles in one day.

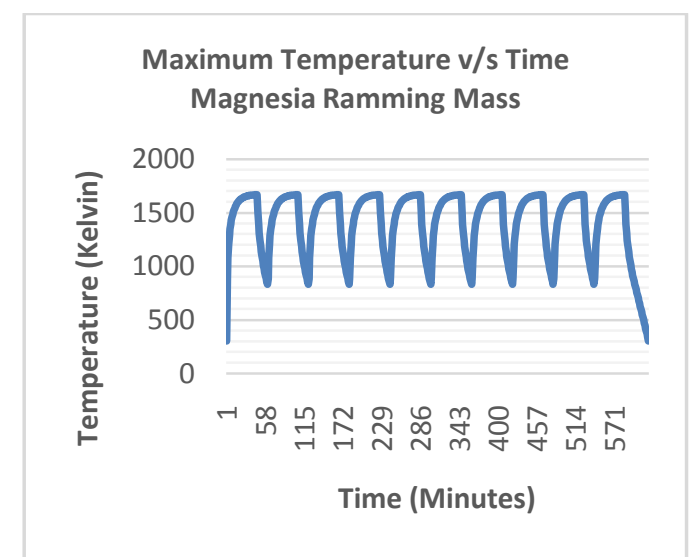

Fig.2 Maximum temperature v/s time graph for magnesia ramming mass 


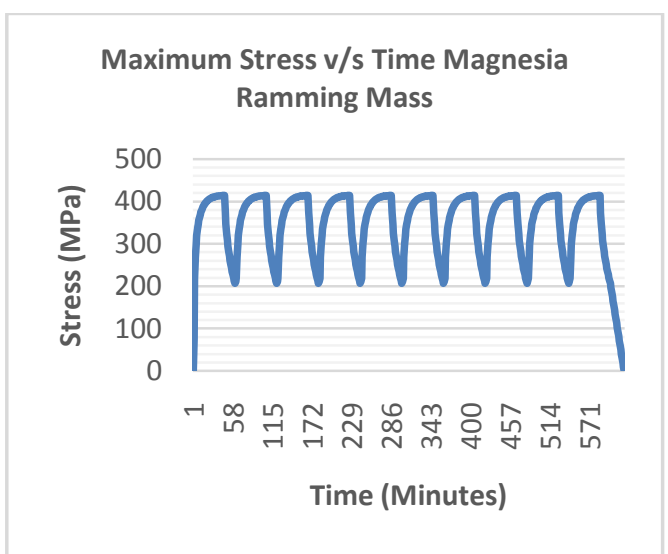

Fig.3 Maximum thermal stress v/s time graph for magnesia ramming mass

We can see from the Fig. 3 that maximum thermal stress is increasing from initial condition $0 \mathrm{MPa}$ and reaches to maximum stress $414 \mathrm{MPa}$ in 45 minutes and then starts reducing and reaches to $206 \mathrm{MPa}$ in next 15 minutes. It again starts increasing and reaches to maximum stress $414 \mathrm{MPa}$ after 105 minutes and again it starts reducing. There are 10 similar thermal stress cycles in one day.

\section{Stress-Life Method}

To determine life of any component by Stress-Life Method, we need to find out ultimate strength and endurance limit of the component for the required material.

We know the values of ultimate stress for these all materials. Magnesia ramming mass is having ultimate strength of $600 \mathrm{MPa}$. We can find out Se' from the equation given below or we can say dividing value of ultimate strength.

We know value of ultimate stress of magnesia ramming mass is $600 \mathrm{Mpa}$.

We know the relation between Sut and Se' so that we can find out Se'.

$\mathrm{Se}^{\prime}=0.5 * \mathrm{Sut}=300 \mathrm{MPa}$

\section{Endurance Limit Modifying Factors}

We have seen that the rotating-beam specimen used in the laboratory to determine endurance limits is prepared very carefully and tested under closely controlled conditions.

It is unrealistic to expect the endurance limit of a mechanical or structural member to match the values obtained in the laboratory.

\section{Some differences include}

Material: composition, basis of failure, variability

Manufacturing: method, heat treatment, fretting corrosion, surface condition, stress concentration

Environment: corrosion, temperature, stress state, relaxation times

Design: size, shape, life, stress state, stress concentration, speed, fretting, galling

Marin identified factors that quantified the effects of surface condition, size, loading, temperature, and miscellaneous items. The question of whether to adjust the endurance limit by subtractive corrections or multiplicative corrections was resolved by an extensive statistical analysis of a 4340 (electric furnace, aircraft quality) steel, in which a correlation coefficient of 0.85 was found for the multiplicative form and 0.40 for the additive form.

A Marin equation is therefore written as

$\mathrm{Se}=\mathrm{ka} * \mathrm{~kb} * \mathrm{kc} * \mathrm{kd} * \mathrm{ke} * \mathrm{kf} * \mathrm{Se}$ '

Where,

$k a=$ surface condition modification factor $=0.84$

$k b=$ size modification factor $=0.98$

$k c=$ load modification factor $=0.96$

$k d=$ temperature modification factor $=0.45$ 
$k e=$ reliability factor $=0.9$

$k f=$ miscellaneous-effects modification factor $=0.95$

$S \mathrm{e}$ '= specimen endurance limit

$\mathrm{Se}=$ endurance limit at the critical location of a machine part in the geometry and condition of use.

We can find out different factor like surface finish factor, size factor, loading factor, temperature factor, reliability factor, miscellaneous effects factor as per the guideline. (Joseph E. Shigleyet al, Machine Engineering Design)

Now, we can find out endurance limit for all different materials.

$\mathrm{Se}=\mathrm{ka} * \mathrm{~kb} * \mathrm{kc} * \mathrm{kd} * \mathrm{ke} * \mathrm{kf} * \mathrm{Se}^{\prime}=100 \mathrm{MPa}$

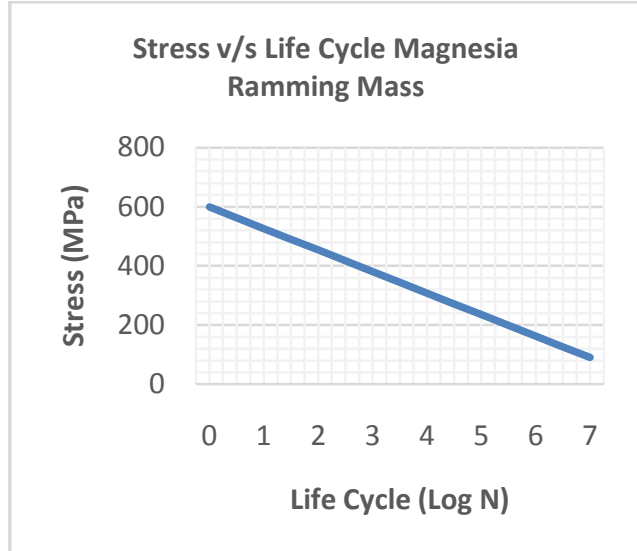

Fig. 3 Stress v/s Life Cycle for Magnesia Ramming Mass

$\mathrm{N}$ (No. of working cycles) $=\mathrm{L}_{\min } \mathrm{X}(100-$ Probability $) /(100 \mathrm{X}$ Miscellaneous Factor $)$

$\mathrm{D}($ No. of working days $)=\mathrm{N} /($ No. of working cycles per day $)$

Table 2 Number of Working Cycles and Number of Working Days with Probability

\begin{tabular}{|l|l|l|l|}
\hline $\begin{array}{l}\text { Sr. } \\
\text { No. }\end{array}$ & $\begin{array}{l}\text { Probability } \\
(\%)\end{array}$ & $\begin{array}{l}\text { No. of working cycles } \\
\text { for Magnesia } \\
\text { Ramming Mass }\end{array}$ & $\begin{array}{l}\text { No. of days } \\
\text { working }\end{array}$ \\
\hline 1 & 100 & 356 & 39.6 \\
\hline 2 & 90 & 363 & 40.3 \\
\hline 3 & 80 & 370 & 41.1 \\
\hline 4 & 70 & 377 & 41.9 \\
\hline 5 & 60 & 384 & 42.7 \\
\hline 6 & 50 & 392 & 43.5 \\
\hline 7 & 40 & 399 & 44.3 \\
\hline 8 & 30 & 406 & 45.1 \\
\hline 9 & 20 & 413 & 45.9 \\
\hline 10 & 10 & 420 & 46.7 \\
\hline
\end{tabular}

We can understand from this table that all cases of induction furnace wall will work for minimum 356 working cycles and 39.6 days. The probability will be decreasing continuously with increase in life span and working days. It happens very rarely that induction furnace wall will work for 420 working cycles and 46.7 days so its probability is minimum $10 \%$ of cases can be having such a large life span.

\section{Conclusion}

Induction Melting Furnaces are highly used now- a-days for melting of different kinds of materials. The problem comes from the magnesia ramming mass of losing its material properties and failure occurs within 350-380 hours of lifetime. It will disturb production schedule as it requires time to replace the induction melting furnace wall of magnesia ramming mass. Explicit finite difference analysis is done for induction melting furnace refractory wall and validation is done with respect to experimental Results. Explicit finite difference analysis is done with respect to actual working conditions of induction furnace and material properties of magnesia ramming mass. Then $\mathrm{S}-\log \mathrm{N}$ Curves are plotted for Life Span Prediction.

We had found life span of different materials like magnesia ramming mass is $350-380$ cycles. From the results of experimental study and explicit finite difference analysis of thermal fatigue failure of induction melting furnace wall, it can be seen that finite difference model exactly predicts the failure of the induction 
furnace refractory wall and the definite solution conditions in the finite difference numerical calculation are accurate. The fatigue life of the induction melting furnace refractory wall under thermal fatigue working conditions was predicted using stress-life method by plotting $\mathrm{S}-\log \mathrm{N}$ curves for magnesia ramming mass on the basis of explicit finite difference calculations and maximum thermal stress in the induction melting furnace refractory wall. We can use it as a linear to increase lifespan or we can use premixed magnesia ramming mass for economical and better working lifespan of induction melting furnace wall.

The accuracy of the fatigue life prediction for the induction melting furnace refractory wall depends upon temperature and thermal stress spectrum calculated at the critical point by explicit finite difference method and $\mathrm{S}-\log \mathrm{N}$ curves prepared from the material properties and boundary condition for magnesia ramming mass.

\section{References}

[1] Nirajkumar Mehta, (May 2012), Review on Computational Investigation on Different Kinds of Furnaces, International Conference on Emerging Technologies and Applications in Engineering, Technology and Sciences, Volume 3, pp 1-7.

[2] N C Mehta, Vipul B Gondaliya, Jayesh V Gundaniya, (February 2013), Applications of Different Numerical Methods in Heat Transfer - A Review, International Journal of Emerging Technology and Advanced Engineering, Volume 3, Issue 2, pp 363 - 368.

[3] N C Mehta, Akash D Raiyani, Vikas R Gondalia, (February 2013), Thermal Fatigue Analysis of Induction Melting Furnace Wall for Silica ramming mass, International Journal of Emerging Technology and Advanced Engineering, Volume 3, Issue 2, pp 357 362 .

[4] Vimal R Nakum, Kevin M Vyas, Niraj C Mehta, (April 2013), Research on Induction Heating - A Review, International Journal of Science and Engineering Applications, Volume 2, Issue 6, pp 141 - 144, DOI: 10.7753/IJSEA0206.1005.

[5] N C Mehta, Viral V Shiyani, Jemish R Nasit, (May 2013), Metal Forming Analysis, International Journal of Emerging Technology and Advanced Engineering, Volume 3, Issue 5, pp 190 - 196.

[6] Vipul Gondaliya, Mehul Pujara, Nirajkumar Mehta, (August 2013), Transient Heat Transfer Analysis of Induction Furnace by Using Finite Element Analysis, International Journal of Applied Research, Volume 3, Issue 8, pp 231 - 234.

[7] N C Mehta, Vasim G Machhar, Ravi K Popat, (October 2013), Thermal Fatigue Analysis of Induction Furnace Wall for Alumina ramming mass, International Journal of Science and Engineering Applications, Volume 2, Issue 10, pp 186 - 190, DOI: 10.7753/IJSEA0210.1002

[8] Akash D Raiyani, N R Sheth, Niraj C Mehta, (July 2014), Thermal Analysis of Hot Wall Condenser for Domestic Refrigerator, International Journal of Science and Research, Volume 3, Issue 7, pp $622-626$

[9] Nirajkumar C Mehta, Dipesh D Shukla, Ravi K Popat, (December 2014), Optimisation of Wall Thickness for Minimum Heat Loss for Induction Furnace by FEA, Indian Foundry Journal, Volume 60, No. 12, pp 19-25.

[10] Nirajkumar C Mehta, Dr.Dipesh D Shukla, Vishvash B Rajyaguru, (April 2015), Numerical Analysis of Furnace: Review, National Conference on Recent Research and Development in Core Disciplines of Engineering, Vadodara, Volume: 2 , pp 1 -7.

[11] Nirajkumar C Mehta, Dr.Dipesh D Shukla, Vishvash B Rajyaguru, (April 2015), Thermal Fatigue Analysis of Induction Furnace Wall for Zirconia, National Conference on Recent Research and Development in Core Disciplines of Engineering, Vadodara, Volume: 2, pp $1-6$.

[12] Nirajkumar C Mehta, Dr.Dipesh D Shukla, Pragnesh D Kandoliya, (April 2015), Comparison of Finite Difference Method and Finite Element Method for 2 D Transient Heat Transfer Problem, National Conference on Recent Research and Development in Core Disciplines of Engineering, Vadodara, Volume: 2, pp 1 - 10

[13] Nirajkumar C Mehta, Dr.Dipesh D Shukla, (June 2015), Thermal Fatigue Analysis of Induction Furnace Wall for Magnesia Ramming Mass, ASME 2015 Applied Mechanics and Materials Conference, At Seattle, Washington, United States of America, Volume: 12 , pp $1-6$.

[14] Nirajkumar C Mehta, Dr.Dipesh D Shukla, Pragnesh D Kandoliya, (December 2016), Advanced Mathematical Modeling of Heat Transfer in Induction Furnace Wall of Zirconia, International Journal of Engineering Research and Technology, Volume 5, Issue 10, pp 176 - 181, DOI: 10.17577/IJERTV5IS120128

[15] Nirajkumar C Mehta, Dr.Dipesh D Shukla, Pragnesh D Kandoliya, (December 2016), Advanced Heat Transfer Analysis of Magnesia Based Refractory Wall of Induction Furnace, National Conference on Emerging Trends in Engineering, Volume 1, pp 1 6.

[16] A V K Suryanarayana, Fuels Furnaces Refractory and Pyrometry, B S Publications

[17] Heat and Mass Transfer A practical Approach, by Yunus A Cengel

[18] Machine Engineering Design, by Joseph E. Shigley and Charles R. Mischke, TATA McGRAW HILL Publication, sixth edition

[19] John Campbell, Castings, (2nd Edition) 